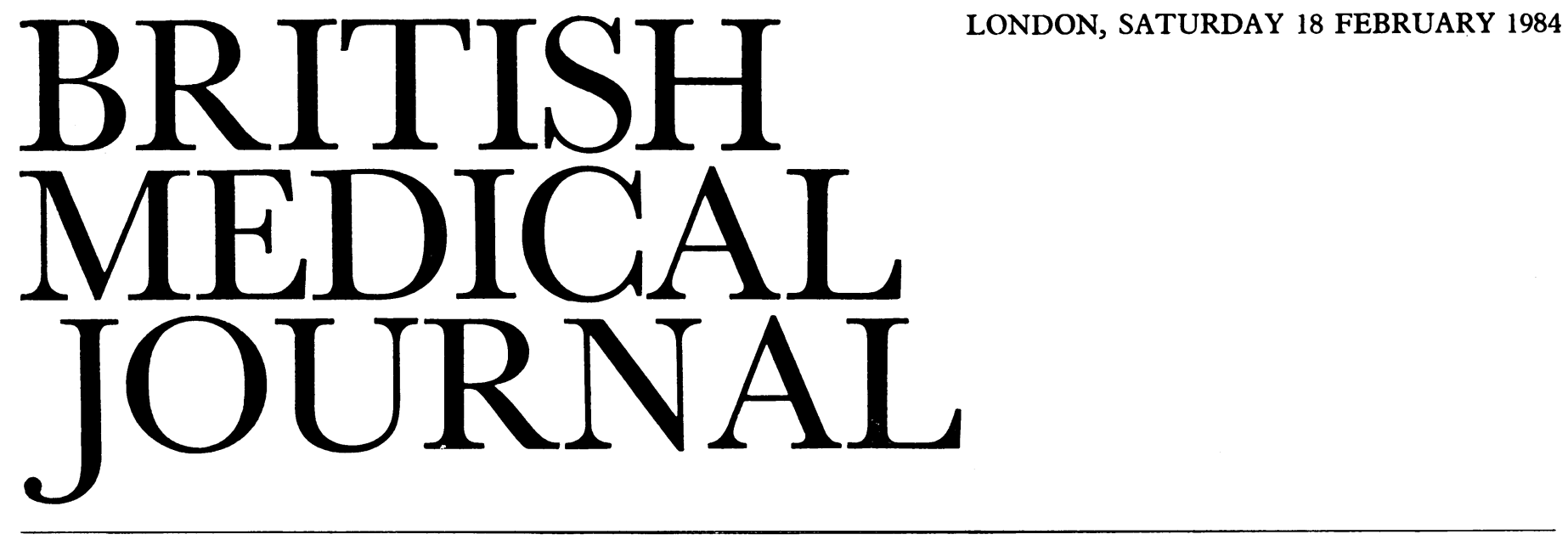

\title{
University cuts, medicine, and the public interest
}

Britain's universities are in rough water. To some extent faculties of medicine have been sheltered, but even their public funding has fallen in real terms by $10-15 \%$ in three years. The immediate future offers no hope of calm-indeed, university grants seem certain to fall behind inflation. Against this background the Secretary of State for Education has asked how universities would cope if their studeni intake were to remain constant but their income were to fall progressively by a further $10-15 \%$ per student over the next decade.

This question, speculative but with a sting in its tail, is not about the number of students (medical or not) but about resources per student. Only last year the Secretary of State for Health confirmed his commitment to the present annual intake target for Great Britain excluding Northern Ireland of 4080 medical students (not all of whom of course qualify). If, as there may be, there is a shortage of jobs for doctors in Britain in the future (but there is no conclusive evidence yet that there will be) the answer does not lie in a shortsighted reduction in our own graduates. The correct solution, much more difficult in terms of professional politics on the one hand and national policy on the other, requires vision and couragevision to reform the steeply pyramidal career structure in the National Health Service; courage to limit medical immigration.

So far university cuts have had several bad and a few arguably good effects on medical education. On the bad side staff:student ratios have fallen in a discipline which more than most needs small group teaching, especially in the clinical years. The new medical schools have not been able to develop academically to their full extent. The universities' provision of basic research facilities has fallen to a point from which it is very difficult to develop projects to the degree of promise sufficient to raise support from outside. Research is also being forced to take third place against the more immediate demands of patients and teaching. ${ }^{1}$ The proper development of smaller departments such as community medicine, general practice, psychiatry, and bioengineering has stopped and at least one department has disappeared altogether. Indiscriminate freezing of vacant posts and early retirements have distorted the relative strengths of departments in a haphazard way.

By contrast, the benefits of hard times have been painful but real. Individual departments have been forced both to look critically at their effectiveness and to redouble their efforts to obtain outside research funds. Institutions have been driven to examine the efficiency of their own operation. London University (which trains one third of the doctors in Britain) has been stimulated to regroup its medical schools and postgraduate institutes into an academically stronger and economically sounder organisation. Some universities have started to direct relatively more funds towards schools or faculties which are attracting most support from research councils. "New blood" lectureships are being created in open, national competition in those departments with most to offer talented younger men and women caught in a trap of diminishing academic opportunity.

Nevertheless, university medical education is now stretched to the limit $^{2}$; does it matter if it snaps? The Royal Commission on University Education in London in 1913 stated that "the main justification for providing adequately for university education is the public interest, and this is perhaps more apparent in the case of medical education than in any other department of learning." 3

There are at least four reasons why it is very much in the public interest for academic medicine not only to survive but to prosper: the fundamental importance both to patients and to the Health Service itself of a constructively critical spirit; the need to continue to produce doctors with wide experience and competence, able to see the general woods for the specialist trees; the practical value of research applied particularly to the care of patients; and the need to combine the resources of the NHS and the universities in the development of the best in all respects that the world can offer.

Academic departments exist not only to plan and coordinate teaching but also to question and to innovate, to disturb, and to extend knowledge and practice. Standards of care will continue to improve only if doctors grow up dissatisfied with current standards of knowledge and service, constructively and relentlessly setting higher standards for themselves, administrators, and politicians. Personal participation of teachers in research is important both in generating this critical spirit and in providing a springboard to deeper knowledge and better service.

Universities are often equated with high technology and remoteness from reality. Paradoxically perhaps the most fundamental contribution of university departments of medicine is their emphasis on the importance of doing comparatively simple things well. It is the acquisition of the skills of listening to and examining patients and the attitude which these skills create of putting the patient at the centre 
of medicine which are currently most threatened. These basic skills are acquired from many hours of painstaking instruction and practice coordinated by and largely given in the first, formative months by clinical academic staff.

This very British approach is unfashionable in many other countries where doctors qualify with little personal, practical knowledge of patients. There, patients are investigation fodder: looking and listening are subsidiary to a succession of unpleasant, often unnecessary, and usually expensive investigations; relations between doctors and patients are correspondingly distant.

Clinical academics are fast becoming the last of the generalists in the main university hospitals. ${ }^{4}$ Not only are they important in the teaching of clinical skills but they also strongly represent the integrating value of experience, interest, and ability across the specialties. Further, they stimulate the curiosity and command the facilities necessary to further knowledge of their subject in interdisciplinary research.

A wider view has never been more important, especially as a foundation on which to base the flexibility necessary to adapt to changing demands of medical practice and social environment. Medical education may have been slow to direct sufficient attention to the non-complex and insubstantial (but not inconsequential) needs of most patients, but the challenge is slowly but surely being grasped. Universities are also adopting a wider orientation of physical and human sciences in relation to their applications to clinical medicine in the early years of the undergraduate course. If universities do not continue to integrate science and medicine, an integration expansive in subjects and expensive in teacher time, who will? And how would retrenchment in university medical education be viewed by the General Medical Council, which is responsible for standards of medical education?

Clinical academic departments also have a special opportunity to find tomorrow's teachers and research workers and to fashion suitable paths for their training. Clinical professors could do this much better if only they had at their disposal career development awards to back winners. Far too many highly talented doctors are allowed to drift by chance into jobs which others could do almost as well while their special abilities are badly needed in the amalgam of teaching, service, and research which is academic medicine.

Critical attitudes arising out of the time spent by teachers in research are important, but so too is the research in its own right. Worthwhile research is impossible without time; the recent reductions in medical academic staffing have seriously eroded that time. ${ }^{5}$ If universities pulled out of clinical research medically relevant research would still continue in science departments, research institutions, and pharmaceutical companies. But clinical academics are uniquely placed to recognise and to investigate the problems which arise day by day in ward and clinic. The sort of research which would suffer is, to take just one example, research into the understanding of the genetic basis of disease, its early diagnosis, and its possible prevention. Currently advances are being made which depend on contact with patients either by those exceptional persons who are both excellent clinicians and scientists or by the conjunction of academic clinician and biochemist in departments situated side by side at the clinical coalface.

The cost of giving up clinical research would, however, be greater than this. Enterprise and inquiry cannot be stifled, and good clinical scientists active in research, teaching, and looking after patients in university hospitals would go elsewhere; medical emigration in general may, be at an end, but high academic ability is still a readily marketable commodity.

Research also brings new technology to the bedside. This is expensive and understandably provokes envy. The NHS must, however, be able to offer the best. Highly technical and expensive services need not be developed in every district general hospital, but development makes great sense where university and NHS resources converge. Special development of university hospitals inevitably creates an unequal demand for resources, but members of health authorities must, as Dainton has pointed out, "appreciate to the full the overriding importance for the future of the Health Service of making the teaching hospitals exemplars of the highest standards of patient care, the best techniques of diagnosis and treatment so that the student doctors are set targets of high commitment and personal performance which they must strive to maintain throughout their later careers." 6

University departments of medicine benefit the NHS in these and other ways. In return NHS doctors make a very substantial contribution to clinical education but their contribution is now seriously threatened by proposed cuts in medical staffing especially in senior registrars in general medicine and surgery, the core clinical subjects in undergraduate medical education. If NHS staff were asked to take over the current teaching and service responsibilities of clinical academic staff they would need more time-time which many could not give, some would not wish to give, and which would have to be paid for. The NHS is itself under siege and there is no realistic possibility of it being able to provide the resources and facilities for teaching and research currently provided by the universities, or of the NHS being able to take over clinical services manned by university paid clinical staff. Universities and the NHS have built up together at university hospitals a standard of education and service in both everyday care and special skill second to none. Service to patients, medical education, and research would all suffer if clinical academic departments collapsed. Unfortunately DHSS moves to reduce the acute services provided by some university teaching hospitals also threaten to undermine this joint achievement.

If the funding per university student falls further and medicine is not protected as a "scientific and vocationally relevant study" faculties of medicine will either have to reduce staff or secure private money. Further reduction of staff would make it impossible for most medical schools to maintain a full range of academic departments without amalgamation, but the marriages which are both geographically feasible and rationally justified are already under way. The only viable alternatives if funding falls further are either to prune the largest schools, to close one or more of the others, or both.

Research councils and private trusts which support research will neither be able nor prepared to support the educational role of universities. Economic fees from private students would seem to be the only practicable alternative. These could be overseas students contracted to return home on qualification so as not to exceed DHSS targets. Ideally most would be on scholarships. Unfortunately, rather than consider such a contractual obligation, the DHSS insists on a limit of 200 new overseas medical students a year-a sad decision both for international relations and for universities prepared to stretch their current facilities in order to pay their way.

Partnership between the universities and the NHS in undergraduate and postgraduate medical education has served the public interest well and promises to provide excellent doctors for the NHS of tomorrow. The public needs to 
guard its interests very jealously in the current debate on university funding, and nowhere more energetically than in medical education.

Dean and Professor of Medicine,

St Mary's Hospital Medical School,

London W2 1 PG

1 Association of Professors of Obstetrics and Gynaecology. The effects of the present financial crisis on academic departments of obstetrics and gynaecology. Br Med f 1983;287:771-4.

${ }^{2}$ Kessel N. Academic medicine in peril. Lancet 1983;ii:899-901.

${ }^{3}$ Royal Commission on University Education in London. Final report. London: HMSO, 1913:para 301. (Cmd 6717.)

${ }^{4}$ Peart WS. Rebirth of the professor of medicine. Lancet 1983; : $: 810-2$.

s Mitchell JRA. The unkindest cut of all: a view of the UGC cuts from Britain's first 20th-century medical school. Lancet 1982;ii:540-5.

${ }^{6}$ Dainton F. Reflections on the universities and the National Health Service. London: Nuffield Provincial Hospitals Trust, 1983:152.

\section{Are antithyroid drugs immunosuppressive?}

Graves' disease is an autoimmune disorder in which hyperthyroidism results from thyroid stimulating antibodies binding to the thyroid cell membrane and stimulating thyroid hormone biosynthesis. Two papers in this issue present new data on the mechanism of action of antithyroid drugs in Graves' disease (pp 518 and 526). The authors claim that as well as blocking the synthesis of thyroid hormone these drugs suppress production of the pathogenic antibodies, and they discuss the mechanisms which may bring about this reduction. The view that antithyroid drugs are immunosuppressive is controversial, and the evidence deserves careful review.

Antithyroid drugs inhibit iodine organification but have no direct effect on the concentration of inorganic iodide by the thyroid. The drugs include the thiouracil drugs and carbimazole, which is rapidly converted to its active metabolite methimazole, and depend for their activity on a thiocarbamide group $(\mathrm{S}=\mathrm{C}-\mathrm{N})$. They bind to thyroid peroxidase competitively with iodide, so inhibiting the oxidation of iodide as well as other reactions catalysed by the enzyme, including the iodination of tyrosyl residues in thyroglobulin and the coupling to form iodothyronines. ${ }^{1-3}$

Doctors have long recognised that in about a third of patients with Graves' hyperthyroidism the disease remits spontaneously. ${ }^{45}$ The first fundamental question, therefore, is whether carbimazole influences the remission rate. If it merely suppresses thyroid hormone synthesis until such time as remission occurs spontaneously then the remission rate will not have changed much since antithyroid drugs were introduced in about 1944. Furthermore, if antithyroid drugs do not affect the underlying disease then the dose and the duration of treatment would be unlikely to influence remission rates. Many series of patients treated with antithyroid drugs have been reported with remission rates varying from $30 \%$ to $75 \% \%^{6.11}$ Obviously the figures depend on how remission is defined; their interpretation must take into account the fluctuating course of Graves' disease ${ }^{12}$ and also other factors such as iodine intake that influence the outcome. ${ }^{1314}$ The remission rate is probably lower in those treated with propranolol alone than in those treated with antithyroid drugs, despite the fact that milder cases are selected for propranolol treatment. ${ }^{15-17}$ The duration of antithyroid treatment varies from one to two years in most series. When treatment is prolonged beyond this the remission rate improves, ${ }^{1018}$ whereas in a study of short term treatment (mean 4.8 months) the remission rate was $29 \% \cdot{ }^{19} \mathrm{~A}$ study of relapse rates also found an association with duration of treatment, varying from $49 \%$ to $91 \%$ after five years. ${ }^{20}$ The remission rate may also be improved if the dose is increased, thus in a comparison of $60 \mathrm{mg}$ methimazole daily with $14 \mathrm{mg}$ daily for $13-15$ months the remission rates were $75 \%$ and $42 \%$ respectively. ${ }^{21}$ Further evidence comes from studies of technetium uptake during treatment: technetium uptake reflects iodine trapping, and antithyroid drugs have no direct effect on trapping, yet a progressive decline in technetium uptake was seen during treatment with carbimazole, indicating an effect at a stage before thyroid hormone biosynthesis. ${ }^{22}{ }^{23}$ Although these data do not prove conclusively that antithyroid drugs influence the course of the disease, they are highly suggestive.

Next we must consider effects on thyroid lymphocytes, serum antibody titres, and $\mathrm{T}$ cell activity in patients receiving these drugs. Michie et al first suggested that the drugs might act on lymphoid tissue and autoantibody production when they observed a decrease in the size of the thymus in patients treated preoperatively with carbimazole. ${ }^{24}$ Later they showed that the thymus involution and decreased lymphocytic infiltration of the thyroid that were seen during treatment with carbimazole were not seen in patients treated with propranolol. ${ }^{25}$ This led them to postulate that the drugs had an immunosuppressive effect, and they also suggested that investigating the pharmacological effects of carbimazole might lead to a better understanding of the pathogenesis of the disease. This we are now beginning to see. Since these observations evidence has mounted that antithyroid drugs block autoantibody production. Falling titres of long acting thyroid stimulator, ${ }^{26}$ thyrotrophin binding inhibiting immunoglobulin, ${ }^{27-29}$ thyroid stimulating antibody, ${ }^{30}$ and microsomal antibody ${ }^{29}$ have been reported during treatment. Also in an animal model of experimental autoimmune thyroiditis the severity of the thyroiditis in euthyroid rats was decreased by pretreatment with methimazole, and this was accompanied by a fall in thyroglobulin antibody titre..$^{31} \mathrm{~T}$ cell activity is also influenced by antithyroid drugs, as was first shown using tritiated thymidine uptake by peripheral blood lymphocytes. ${ }^{32}$ More recently several groups using OKT8 mouse monoclonal antibody to distinguish suppressor/cytotoxic $\mathrm{T}$ cells have shown a decrease in the number of $T$ suppressor cells in untreated patients and also in their activity. ${ }^{33}$ This finding has been extended by Ludgate et al (p 526), who have found in addition an increase in activated $T$ cells and a return of these $\mathrm{T}$ cell abnormalities to normal during six months of treatment with carbimazole.

These effects on lymphocytes could be secondary to the decreased thyroid hormone synthesis rather than a direct effect. In vitro studies are therefore important. McGregor et al cultured in vitro peripheral blood lymphocytes from patients with Hashimoto's disease and found that IgG synthesis and thyroid antibody production were inhibited in the presence of methimazole $10 \mu \mathrm{mol} / \mathrm{l}$ but not propranolol. ${ }^{29}$ These results were confirmed in a second study, but a higher concentration $(100 \mu \mathrm{mol} / \mathrm{l})$ of methimazole was needed. ${ }^{34}$ Thyroid hormones did not influence these results. Most of the thyroid antibodies are probably produced by lymphocytes within the thyroid, so the drug concentration achieved in the thyroid is more important than the blood concentration. Only recently has it been possible to measure the thyroid concentration in patients, and the results suggest that the thyroidal concentrations of methimazole may be less than those reported to be immunosuppressive. There was also no correlation 